\title{
Non-Invasive Laser Modalities in Treatment of Posterior Ventral Infarction
}

\author{
Jackson S Pollock ${ }^{*}$, Rosa Q Luxemburg ${ }^{1}$, Hercule CM Poirot ${ }^{2}$ and Friedrich X Engels ${ }^{3}$ \\ ${ }^{1}$ Brockton State University, USA \\ ${ }^{2}$ U.S. National Clinics of Health, USA \\ ${ }^{3}$ Massapequa University, USA
}

\section{Background}

Postoperative myocardial infarction is not a well-studied topic after lung surgery. From the few reports in the literature, the frequency to range between $0.7 \%$ to $2 \%$, but it is difficult to determine an exact standardized incidence, in part due to differences in postoperative presentation, differences in EKG interpretation and a variation in the choice of serum cardiac markers.

Both atmospheric pressure and partial pressure of oxygen fall even with a modest rise in altitude [1] and this may increase PVR. The fall in atmospheric pressure is nearly linear within the range of altitudes that is relevant to most people (below 5000 metres), with a fall rate of $1 \%$ to $1.5 \%$ per 100 metres altitude above sea level. Therefore, even at the modest altitude of 500 metres, atmospheric pressure is $6.5 \%$ lower than it is at sea level. This reduction in atmospheric pressure results in a parallel reduction in the partial pressure of oxygen, since the proportion of oxygen in the atmosphere is constant $(21 \%)$ up to an altitude of 110000 metres [2]. Thus, at 500 metres the partial pressure of oxygen is also $6.5 \%$ lower than it is at sea level. Such a fall in oxygen levels may be sufficient to increase PVR. This is in keeping with our data, which suggests that the rate of early Fontan failure may increase at even modest altitudes, with a Spearman rank correlation of 0.74 ( $p=$ 0.001 ). The failure of logistic regression to account for all the non-random variation in failure rate is almost certainly due to factors beyond the scope of this study, such as individual surgeon and unit performance and variation in patient characteristics.

\section{Methods}

The MxPG across the prosthesis was estimated by the modified Bernoulli equation $\left(4 \mathrm{~V}^{2}\right)$; the $\mathrm{MnPG}$ was derived by planimetry of the Doppler envelope. Measurements from at least three velocity envelopes were averaged to assure consistency. The EOA was calculated by the continuity equation: EOA $\left(\mathrm{cm}^{2}\right)=$ CSA LVOT $\times$ V LVOT integral/V Trans prosthetic integral, where CSA is cross-sectional area, LVOT is left ventricular outflow tract, and $\mathrm{V}$ is velocity integral. Assuming a circular shape, the CSA LVOT was calculated as: $3.14 \times(D / 2)^{2}$, where $D$ is the inner diameter of the left ventricular outflow tract. The V LVOT was obtained with pulsed-wave Doppler in the left ventricular outflow tract proximal to the aortic prosthesis from the apical five-chamber view; the $V$ Trans prosthetic was obtained with continuous-wave Doppler from the apical five-chamber view [3]. The EOAi was calculated by the formula EOA/BSA (body surface area). LVM was derived from Devereux's formula and LVMi from LVM/BSA. The DVI was calculated by the formula: $V$ integral LVOT/ $V$ integral trans prosthetic (normal value $>0.40$ ). The CO was derived from the formula: Stroke volume $($ CSA $\times$ V LVOT integral) $\times$ HR.

The patient underwent a median sternotomy whereupon a giant fatty tumour was found arising superficially from the medial border of the right lung occupying most of the right chest and extending to the anterior mediastinum. The mass was compressing the right lung with no evidence of local invasion. The right phrenic nerve was identified and preserved, and the tumour was found to be adherent to the medial border of the lung and was easily dissected with sharp dissection and removed en bloc. The postoperative recovery was uneventful, and the patient was discharged home on the seventh postoperative day. Six months postoperatively the patient remains well. The final histology of the tumour, measuring $25.5 \times 17.5 \times 6.5$ $\mathrm{cm}$, and weighing $1134 \mathrm{~g}$ (Figure 1), was a pulmonary hamartoma with predominantly adipose and leiomyomata's differentiation.

\section{Results}

A standard median sternotomy is performed, and the

*Corresponding author: Jackson S Pollock, Brockton State University, USA

Accepted: February 01, 2020

Published online: February 03, 2020

Citation: Pollock JS, Luxemburg RQ, Poirot HCM, et al. (2020) Non-Invasive Laser Modalities in Treatment of Posterior Ventral Infarction. J Cardiothorac Surg Ther 4(1):43-45 

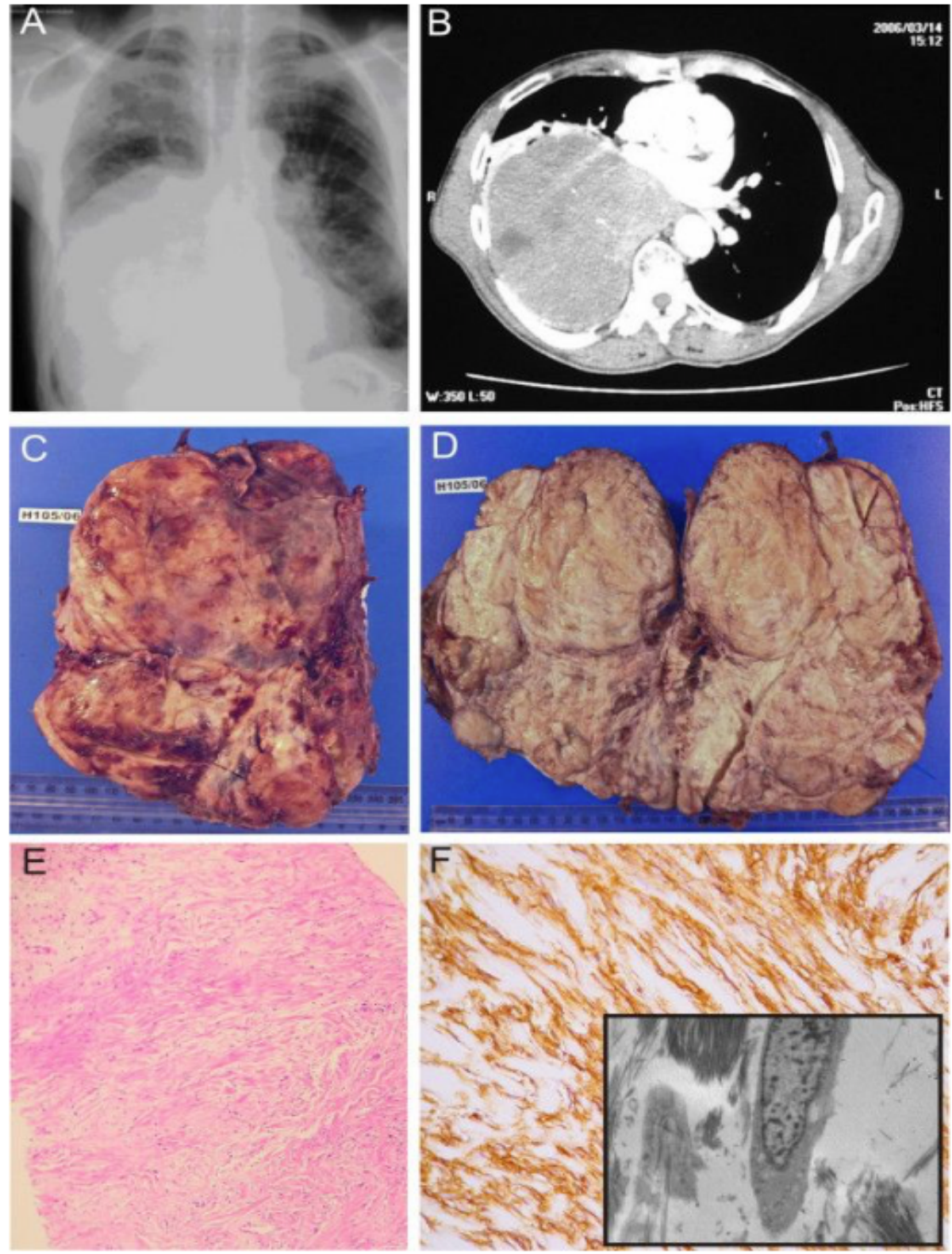

Figure 1: Tumour histology.

thymus gland, if large, is partially removed. The innominate artery is dissected out cephalad to the innominate vein and looped with a vessel loop. This is usually done prior to the opening of the pericardium. A Castaneda clamp is applied and a longitudinal arteriotomy is made. A 3.5 $\mathrm{mm}$ thin-walled PTFE graft is anastomosed end-to-side to the innominate artery using a continuous $7 / 0$ prolene suture. The innominate artery is unclamped, and the graft is flushed and clamped and left as long as possible, usually $15-20 \mathrm{~cm}$. An $8 \mathrm{Fr}$ or $10 \mathrm{Fr}$ ECMO arterial cannula is inserted into the end of the graft, connected to the arterial side of the bypass circuit, and de-aired in the usual fashion. The cardioplegia line is frequently connected to the side-arm of the arterial cannula, obviating the need to place a needle in the ascending aorta for the administration of cardioplegia. The graft, being soft and flexible, easily drapes out of the operative field and is tacked to the drapes where the rigid ECMO cannula joins the graft. The patient is heparinized either before the graft is sutured to the innominate artery or once the anastomosis is completed. The pericardium is then opened and the right atrium or venae cavae are cannulated.

Since physiology is maintained in RAs, the string sign may be a clinically adaptive response to optimize flow characteristics. These grafts respond to endogenous (and exogenous) vasodilators to alter blood flow in high demand situations and also optimize the graft size-match with the target vessel. This balance between endothelium-mediated vasoconstriction and vasodilation was revealed by the investigators in their study. They demonstrated autoregulation of the RA conduit diameter, which adapted to match target vessel size [4]. Also, the investigators demonstrated that endothelial-dependent vasodilation is preserved in the RA at 3-months post-surgery, but not with the LSV 
grafts, such that administration of intravenous nitrate in RA patients with string signs resulted in all having some degree of dilation [5].

\section{Conclusion}

Two patients ( $C$ and $E$ ) produced significant differences in treatment preference between round 1 and round 2 of voting suggesting that the discussion between rounds had produced an overall change in clinical opinion. Both of these patients had significant co-morbidity which may have influenced the choice of treatment with one swinging from predominantly surgery to angioplasty and the other from an equal number of angioplasty and medical to predominantly medical therapy [6]. Overall, comparing the pattern of votes between rounds 1 and 2, there was a small non-significant reduction in the number of votes for medical therapy alone with an increased number of votes for medical therapy followed by either PCl or CABG if symptoms dictated and no significant increase in the overall use of revascularization.

\section{References}

1. Verma S, Szmitko PE, Weisel RD, et al. (2004) Should radial arteries be used routinely for coronary artery bypass grafting? Circulation 110: e40-e46.

2. Gaudino M, Alessandrini F, Pragliola C, et al. (2004) Effect of target artery location and severity of stenosis on mid-term patency of aorta-anastomosed vs. internal thoracic artery-anastomosed radial artery grafts. European Journal of Cardio-thoracic Surgery 25: 424-428.

3. Khot UN, Friedman DT, Pettersson G, et al. (2004) Radial artery bypass grafts have an increased occurrence of angiographically severe stenosis and occlusion compared with left internal mammary arteries and saphenous vein grafts. Circulation 109: 20862091.

4. Desai ND, Cohen EA, Naylor D, et al. (2004) A randomized comparison of radial-artery and saphenous-vein coronary bypass grafts. N Engl J Med 351: 2302-2309.

5. Buxton BF, Raman JS, Ruengsakulrach P, et al. (2003) Radial artery patency and clinical outcomes: five-year interim results of a randomized trial. J Thorac Cardiovasc Surg 125: 1363-1371.

6. Singh RN, Beg RA, Kay EB (1986) Physiological adaptability: the secret of success of the internal mammary artery grafts. Ann Thorac Surg 41: 247-250.

DOI: $10.36959 / 582 / 420$ 